\title{
Risk attitudes over small and large stakes recalibrated
}

\author{
Eduardo Zambrano* \\ February 11, 2019
}

\begin{abstract}
In this paper I provide bounds on the marginal rate of substitution between losing \$ $\mathrm{x}$ and winning \$y, starting from wealth level \$w, for a risk averse individual that rejects a small stake gamble for a range of initial wealth levels. I then prove a theorem that can be used to identify the kinds of large stakes that would be rejected by any such individual. The theorems allow us to understand how much risk aversion is embedded in anyone's rejections of certain small stakes gambles and provide tighter connections between the results in Rabin (2000) and the received theory of decision making under risk.
\end{abstract}

KEYwORDS: Expected Utility Theory, Risk Aversion Calibration. JEL CODE: D81.

\footnotetext{
* Department of Economics, California Polytechnic State University, San Luis Obispo, CA. Email: ezambran@,calpoly.edu. Phone: 805-756-5327.
} 


\section{Introduction}

In 2000, Matthew Rabin proved a theorem stating that if, for some range of initial wealth levels and for some $g>l>0$, a risk averse decision maker rejects a 50-50 lottery of losing $l$ and gaining $g$, then there is an upper bound on the rate at which (Bernoulli) utility increases above a given wealth level, and a lower bound on the rate at which utility decreases below that wealth level. Rabin was then able to produce examples such as the following: "Suppose we knew a risk-averse person turns down 50-50 lose $\$ 100$ /gain $\$ 105$ bets for any lifetime wealth level less than $\$ 350,000$. Then we know that from an initial wealth level of $\$ 340,000$ the person will turn down a 50-50 bet of losing $\$ 4,000$ and gaining $\$ 635,670$." (Rabin 2000, p 1282).

While Rabin did not offer a closed form solution to the problem of how to construct these examples, ${ }^{1}$ he did offer an intuition behind their validity that depends on the rate at which marginal utility of wealth must decline within the domain of wealth levels in which the original small gamble was rejected. The implication of his keen observation is that for a lottery to be accepted in which one obtains either a larger gain, $y$, or a larger loss, $x$, with equal probability, one would need a very large $y$ even for a relatively low $x$.

There is, of course, nothing wrong with the mathematics behind Rabin's examples, but exactly what is going on in them from a behavioral standpoint is not clear because of the reliance of his arguments on whether certain marginal utilities are large or small. This is problematic because modern decision theorists do not treat these marginal utility magnitudes as having observable content.

In this paper I prove a theorem that establishes bounds on observable characteristics of behavior -the marginal rate of substitution between losing $x$ and winning $y$, starting from wealth level $w$ - for an individual that rejects a small stake gamble for a range of initial wealth levels. I am then able to prove a theorem that can identify the kinds of large stakes that would be rejected by any such individual. The results allow us to understand with more precision how much risk aversion is embedded in anyone's rejections of certain small stakes gambles. The methods I use to prove these results are very different to those used by Rabin and are also of independent interest.

While the results in the present paper are relevant for the proper interpretation of Rabin's result, I do not enter this debate here. ${ }^{2}$ My goal is more modest: to better understand the behavioral content behind his result, and to tie it more closely to the received theory of decision making under risk.

\footnotetext{
${ }^{1}$ He does this only for the case in which the small bet is rejected for all wealth levels in a Corollary to his Theorem.

${ }^{2}$ The reader interested in this matter can consult the arguments in Bleichordt et al 2017, Cox and Sadiraj 2006, Cox et al 2013, Palacios-Huerta and Serrano 2006, Rabin and Thaler 2001, Rubinstein 2006 and, of course, Rabin 2000.
} 


\section{Setting}

I consider an individual with wealth level $w>0$ and twice continuously differentiable Bernoulli utility function $u$, strictly increasing and concave. Consider gambles, $\frac{1}{2}(-x)+\frac{1}{2}(y)$, such that, starting from $w$, the decision maker has the opportunity to lose $x$ or gain $y$ with equal probability. Let $x<w$. Then the marginal rate of substitution at $w$ between $x$ and $y$ is given by $\operatorname{MRS}(w-x, w+$ $y)=\frac{u^{\prime}(w-x)}{u^{\prime}(w+y)}$. The individual rejects the gamble $\frac{1}{2}(-x)+\frac{1}{2}(y)$ at wealth level $w$ whenever $\frac{1}{2} u(w+y)+\frac{1}{2} u(w-x)<u(w)$.

\section{Results}

Let $I=[\underline{w}, \bar{w}]$ be a closed interval in the positive real line. Let $g>l>0$.

Theorem 1. Suppose that for every $w \in I$ the individual rejects the gamble $\frac{1}{2}(-l)+\frac{1}{2}(g)$. Then, for every $w \in I$ and every $x, y>0$ with $x<w$,

$$
\operatorname{MRS}(w-x, w+y)>\left(1+a^{*} \min \{x, w-\underline{w}\}\right)\left(1+a^{*} \min \{y, \bar{w}-w\}\right)
$$

where $a^{*}$ is the solution for $a$ to the equation $e^{a l}+e^{-a g}-2=0$.

The implication is that the magnitude of the gain that would compensate the individual for a given loss, in a 50:50 gamble, grows very rapidly with the size of the loss. This is seen in Theorem 2 below.

Theorem 2. Suppose that for every $w \in I$ the individual rejects the gamble $\frac{1}{2}(-l)+\frac{1}{2}(g)$. Then, for all $w \in I$ and $x \in(0, w-\underline{w}]$, the individual rejects the gamble $\frac{1}{2}(-x)+\frac{1}{2} y$, where

$$
y=\left\{\begin{array}{cc}
\frac{1}{a^{*}}\left(e^{\frac{a^{* 2} x^{2}}{2}+a^{*} x}-1\right) & \text { if } x<\bar{x} \\
{\left[\frac{1}{a^{*}}+(\bar{w}-w)\right]\left(\frac{a^{* 2}}{2} x^{2}+a^{*} x\right)} & \text { if } x \geq \bar{x}
\end{array}\right.
$$

$a^{*}$ is as in Theorem $1, \bar{x}=\min \{\ddot{x}, w-\underline{w}\}$ and $\ddot{x}$ is the non-negative solution for $x$ of the expression $\left[\frac{1}{a^{*}}+(\bar{w}-w)\right]\left(\frac{a^{* 2}}{2} x^{2}+a^{*} x\right)=\bar{w}-w$.

When the individual rejects the small gamble $\frac{1}{2}(-l)+\frac{1}{2}(g)$ at all wealth levels the theorem simplifies further, as shown in the Corollary below. 
Corollary. Suppose that for every $w>0$ the individual rejects the gamble $\frac{1}{2}(-x)+\frac{1}{2}(y)$. Then, for all $w>0$ and $x \in(0, w]$ the individual rejects the gamble $\frac{1}{2}(-x)+\frac{1}{2}(y)$, where

$$
y=\frac{1}{a^{*}}\left(e^{\frac{a^{* 2} x^{2}}{2}+a^{*} x}-1\right)
$$

and $a^{*}$ is as in Theorem 1 .

\section{Remarks}

\section{a. Illustrating the content of Theorems 1 and 2}

Consider the case of Tito, who rejects the gamble $\frac{1}{2}(-\$ 100)+\frac{1}{2}(\$ 120)$ for all wealth levels up to $\$ 500,000$. Then, for $w=\$ 300,000$ and $0<x \leq \$ 300,000$, Tito rejects the gamble $\frac{1}{2}(-x)+\frac{1}{2} y$, where

$$
y=\left\{\begin{array}{cc}
602\left(e^{\left(\frac{x^{2}}{2 \cdot 602^{2}}+\frac{x}{602}\right)}-1\right) & \text { if } x<439 \\
\left(0.28 x^{2}+333 x\right) & \text { if } x \geq 439
\end{array}\right.
$$

This implies that Tito will reject the gamble $\frac{1}{2}(-\$ 10$ thousand $)+$ $\frac{1}{2}$ (\$31 million) at wealth level $\$ 300,000$. This is because the marginal rate of substitution between losing $x$ and gaining $y$ with equal probability from $w=$ $\$ 300,000$ grows extremely rapidly from its initial value of 1 (when $x=y=0$ ). Table 1 illustrates these facts.

\begin{tabular}{lll}
\hline When $x$ is & $\begin{array}{l}\text { Tito rejects the gamble } \\
\frac{1}{2}(-x)+\frac{1}{2}(y),\end{array}$ & $\begin{array}{l}\text { and } M R S(w-x, w+y) \\
\text { would have to be at least } \\
\text { where } y \text { is }\end{array}$ \\
\hline$\$ 200$ & $\$ 285$ & 1.96 \\
$\$ 1,000$ & $\$ 610,463$ & 888 \\
$\$ 10,000$ & $\$ 31,040,051$ & 5875 \\
\hline
\end{tabular}

Table 1. Tito rejects a $50-50$ lose $\$ 100 /$ gain $\$ 120$ for all wealth levels up to $\$ 500,000$. The table reports, for $w=\$ 300,000$, some gambles Tito will reject, and corresponding lower bounds on the marginal rates of substitution between $x$ and $y$ when holding the gamble $\frac{1}{2}(-x)+\frac{1}{2}(y)$ at wealth level $w=\$ 300,000$.

\section{b. Illustrating the content of the Corollary}

Consider now the case of Carly, who rejects the gamble $\frac{1}{2}(-\$ 100)+\frac{1}{2}(\$ 120)$ for all wealth levels. Then at all wealth levels Carly rejects the gamble $\frac{1}{2}(-x)+\frac{1}{2} y$, 
where $y=602\left(e^{\left(\frac{x}{602}+\frac{x^{2}}{2 \cdot 602^{2}}\right)}-1\right)$. This implies that Carly will reject the gamble $\frac{1}{2}(-\$ 3$ thousand $)+\frac{1}{2}(\$ 22$ billion $)$ at all wealth levels. This is because, as before, the marginal rate of substitution between losing $x$ and gaining $y$ with equal probability at any wealth level grows extremely rapidly from its initial value of 1 (when $x=y=0$ ).

Figure 1 illustrates this fact. The horizontal axis measures the magnitude $w-x$ and the vertical axis measures the magnitude $w+y$, where $w=\$ 300,000$. $^{3}$ The orange line represents the indifference curve that passes through $(w, w)$ for the individual with CARA preferences that is indifferent between taking and not taking the gamble $\frac{1}{2}(-\$ 100)+\frac{1}{2}(\$ 120)$. That individual has coefficient of absolute risk aversion equal to $a^{*}=\frac{1}{602}$. While we don't know that Carly is that risk averse in general, we know that she has to be at least as risk averse as the individual whose indifference curve is represented by the green line, which is a depiction of expression (2) from the Corollary. Both the green and the orange indifference curves shown in the figure have first derivative equal to -1 and second derivative equal to $2 \cdot a^{*}$ when $x=0$. Further, the green indifference curve quickly becomes very steep as $x$ increases in size. To wit: the magnitude of the slope of the green indifference curve at point $\mathrm{A}$, where $x=\$ 600$, is 8.9 , at point $\mathrm{B}$, where $x=\$ 1200$, is 161 , whereas at point $\mathrm{C}$, where $x=\$ 1800$ (not depicted in Figure 1), is 6980 .

\section{[Insert Figure 1 about here]}

\section{c. Stronger theorems}

The theorems in this paper are all weaker than they could be. They have, however, the advantage that they are relatively straightforward to prove, the equations for computing the bounds presented in the theorems all have closed form solutions, and they directly point to how much risk aversion is being implicitly assumed when one postulates that a decision maker rejects a small gamble for a range of wealth levels, or for all wealth levels.

\section{Appendix: Proofs}

The following Lemma will be very useful in what follows:

Lemma. Suppose that for every $w \in I$ the individual rejects the gamble $\frac{1}{2}(-l)+$ $\frac{1}{2}(g)$. Then there is $a^{*}>0$ such that, for every $w_{1}, w_{2} \in I$ with $w_{1}<w_{2}$,

$$
u^{\prime}\left(w_{1}\right)>u^{\prime}\left(w_{2}\right)\left(1+a^{*}\left(w_{2}-w_{1}\right)\right)
$$

where $a^{*}$ is the solution for $a$ to the equation $e^{a l}+e^{-a g}-2=0$.

\footnotetext{
${ }^{3}$ For the purposes of drawing this graph I allow $x$ and $y$ to be negative.
} 
Proof: By the mean value theorem, we have that $u^{\prime}\left(w_{1}\right)=u^{\prime}\left(w_{2}\right)+$ $\left(w_{1}-w_{2}\right) u^{\prime \prime}(\theta)$ for $\theta \in\left(w_{1}, w_{2}\right)$. It then follows that

$$
\begin{gathered}
u^{\prime}\left(w_{1}\right)=u^{\prime}\left(w_{2}\right)\left[1+\left(w_{1}-w_{2}\right) \frac{u^{\prime \prime}(\theta)}{u^{\prime}\left(w_{2}\right)}\right]= \\
u^{\prime}\left(w_{2}\right)\left[1+\left(w_{2}-w_{1}\right)\left(-\frac{u^{\prime \prime}(\theta)}{u^{\prime}\left(w_{2}\right)}\right)\right] \geq u^{\prime}\left(w_{2}\right)\left[1+\left(w_{2}-w_{1}\right)\left(-\frac{u^{\prime \prime}(\theta)}{u^{\prime}(\theta)}\right)\right]
\end{gathered}
$$

where the last inequality follows from the fact that $u^{\prime}(\theta) \geq u^{\prime}\left(w_{2}\right)$ and the concavity of $u$. Then, by the Proposition in Palacios-Huerta and Serrano (2006) there is $a^{*}>0$ such that the Arrow-Pratt coefficient of absolute risk aversion of this individual is greater than $a^{*}$ for $w=\theta$, and where $a^{*}$ is the solution for $a$ to the equation $e^{a l}+e^{-a g}-2=0 .{ }^{4}$ Consequently,

$u^{\prime}\left(w_{1}\right) \geq u^{\prime}\left(w_{2}\right)\left[1+\left(w_{2}-w_{1}\right)\left(-\frac{u^{\prime \prime}(\theta)}{u^{\prime}(\theta)}\right)\right]>u^{\prime}\left(w_{2}\right)\left[1+a^{*}\left(w_{2}-w_{1}\right)\right]$

which is what we wanted to show.

Proof of Theorem 1: Suppose that for every $w \in I$ the individual rejects the gamble $\frac{1}{2}(-l)+\frac{1}{2}(g)$. Fix $w \in I$ and $x, y>0$. Let $a^{*}$ be as in the Lemma. We have to consider four cases.

Case 1: $\underline{w} \leq w-x<w<w+y \leq \bar{w}$. The Lemma implies that $u^{\prime}(w-x)>$ $u^{\prime}(w)\left(1+a^{*} x\right)$ and that $u^{\prime}(w)>u^{\prime}(w+y)\left(1+a^{*} y\right)$. It follows that $u^{\prime}(w-$ $x)>u^{\prime}(w+y)\left(1+a^{*} y\right)\left(1+a^{*} x\right)$, that is, $\frac{u^{\prime}(w-x)}{u^{\prime}(w+y)}>\left(1+a^{*} x\right)\left(1+a^{*} y\right)$.

Case 2: $\quad w-x<\underline{w} \leq w<w+y \leq \bar{w}$. The Lemma implies that $u^{\prime}(\underline{w})>$ $u^{\prime}(w)\left(1+a^{*}(w-\underline{w})\right)$ and that $u^{\prime}(w)>u^{\prime}(w+y)\left(1+a^{*} y\right)$. It follows that $u^{\prime}(w-x)>u^{\prime}(\underline{w})>u^{\prime}(w+y)\left(1+a^{*} y\right)\left(1+a^{*}(w-\underline{w})\right)$, that is, $\frac{u^{\prime}(w-x)}{u^{\prime}(w+y)}>$ $\left(1+a^{*}(w-\underline{w})\right)\left(1+a^{*} y\right)$.

Case 3: $\quad \underline{w} \leq w-x<w \leq \bar{w}<w+y$. Again, the Lemma implies that $u^{\prime}(w-x)>u^{\prime}(w)\left(1+a^{*} x\right)$ and that $u^{\prime}(w)>u^{\prime}(\bar{w})\left(1+a^{*}(\bar{w}-w)\right)$. It follows that $u^{\prime}(w-x)>u^{\prime}(\bar{w})\left(1+a^{*} x\right)\left(1+a^{*}(\bar{w}-w)\right)>u^{\prime}(w+y)(1+$ $\left.a^{*} x\right)\left(1+a^{*}(\bar{w}-w)\right)$, that is, $\frac{u^{\prime}(w-x)}{u^{\prime}(w+y)}>\left(1+a^{*} x\right)\left(1+a^{*}(\bar{w}-w)\right)$.

${ }^{4}$ The reader should note that the Proposition in Palacios-Huerta and Serrano (2006) requires $u$ to satisfy non-increasing absolute risk aversion. The proposition, however, holds without that assumption, as they show in page 254 of their paper. 
Case 4: $\quad w-x<\underline{w} \leq w \leq \bar{w}<w+y$. From the Lemma we obtain that $u^{\prime}(\underline{w})>u^{\prime}(w)\left(1+a^{*}(w-\underline{w})\right)$ and that $u^{\prime}(w)>u^{\prime}(\bar{w})\left(1+a^{*}(\bar{w}-w)\right)$. It follows that $u^{\prime}(w-x)>u^{\prime}(\underline{w})>u^{\prime}(\bar{w})\left(1+a^{*}(w-\underline{w})\right)\left(1+a^{*}(\bar{w}-w)\right)>$ $u^{\prime}(w+y)\left(1+a^{*}(w-\underline{w})\right)\left(1+a^{*}(\bar{w}-w)\right)$, that is, $\frac{u^{\prime}(w-x)}{u^{\prime}(w+y)}>\left(1+a^{*}(w-\right.$ $\underline{w}))\left(1+a^{*}(\bar{w}-w)\right)$.

All these cases can be summarized in the expression

$$
\frac{u^{\prime}(w-x)}{u^{\prime}(w+y)}>\left(1+a^{*} \min \{x, w-\underline{w}\}\right)\left(1+a^{*} \min \{y, \bar{w}-w\}\right)
$$

which is what we wanted to show.

Proof of Theorem 2: Let $w \in I$. Let $y_{w}(x)$ be such that $\frac{1}{2} u\left(w+y_{w}(x)\right)+$ $\frac{1}{2} u(w-x)=u(w)$ for all $x$. Fix $x \in(0, w-w]$.Then, by Theorem 1,

$$
\frac{d y_{w}(x)}{d x}>\left(1+a^{*} x\right)\left(1+a^{*} \min \left\{y_{w}(x), \bar{w}-w\right\}\right)
$$

Therefore, as long as $y_{w}(x) \leq \bar{w}-w$ and $x \in\left(0, w-\underline{w}\right.$, we have that $\frac{d y_{w}(x)}{d x}>$ $\left(1+a^{*} x\right)\left(1+a^{*} y_{w}(x)\right)$, which is a differential inequality with solution

$$
y_{w}(x)>\frac{1}{a^{*}}\left(e^{\frac{a^{2} x^{2}}{2}+a^{*} x}-1\right) \text { for } x \text { such that } 0<x \leq \min \{\dddot{x}, w-\underline{w}\}
$$

and where $\dddot{x}$ is the non-negative solution for $x$ of the expression $f(x):=$ $\frac{1}{a^{*}}\left(e^{\frac{a^{* 2} x^{2}}{2}+a^{*} x}-1\right)=\bar{w}-w$.

On the other hand, as long as $y_{w}(x) \geq \bar{w}-w$ and $x \in(0, w-\underline{w}]$, we have that $\frac{d y_{w}(x)}{d x}>\left(1+a^{*} x\right)\left(1+a^{*}(\bar{w}-w)\right)$, which is a differential inequality with solution $y_{w}(x)>\left[\frac{1}{a^{*}}+(\bar{w}-w)\right]\left(\frac{a^{* 2}}{2} x^{2}+a^{*} x\right)$, for $x$ such that $\ddot{x} \leq x \leq w-\underline{w}$, and where $\ddot{x}$ is the non-negative solution for $x$ of the expression $g(x):=$ $\left[\frac{1}{a^{*}}+(\bar{w}-w)\right]\left(\frac{a^{* 2}}{2} x^{2}+a^{*} x\right)=\bar{w}-w$. Notice that we are not interested in the case in which $\ddot{x}>w-\underline{w}$, as that would require for us to consider values for $x$ greater than $w-\underline{w}$, which contravenes one of the hypotheses of the theorem. 
So far we have shown that $y_{w}(x)>\frac{1}{a^{*}}\left(e^{\frac{a^{*} x^{2}}{2}+a^{*} x}-1\right)$ for $x$ such that $0<x \leq$ $\min \{\dddot{x}, w-\underline{w}\}$, and $y_{w}(x)>\left[\frac{1}{a^{*}}+(\bar{w}-w)\right]\left(\frac{a^{* 2}}{2} x^{2}+a^{*} x\right)$, for $x$ such that $\ddot{x} \leq$ $x \leq w-\underline{w}$. The last step in the proof is to show that $\ddot{x} \leq \dddot{x}$. To see that this is so notice that isolating $\frac{a^{* 2} x^{2}}{2}+a^{*} x$ in the expression $f(x)=y$ leads to $\frac{a^{* 2} x^{2}}{2}+a^{*} x=$ $\ln \left(1+a^{*} y\right)$ whereas isolating $\frac{a^{* 2} x^{2}}{2}+a^{*} x$ in the expression $g(x)=y$ leads to $\frac{a^{* 2} x^{2}}{2}+a^{*} x=\frac{a^{*} y}{1+a^{*}(\bar{w}-w)}$. From these conclusions it follows that $\frac{a^{* 2} \dddot{x}^{2}}{2}+a^{*} \dddot{x}=$ $\ln \left(1+a^{*}(\bar{w}-w)\right)$ and $\frac{a^{* 2} \ddot{x}^{2}}{2}+a^{*} \ddot{x}=\frac{a^{*}(\bar{w}-w)}{1+a^{*}(\bar{w}-w)}$. The fact that $\ln (1+z) \geq \frac{z}{1+z}$ for all $z \geq 0$ implies that $\ln \left(1+a^{*}(\bar{w}-w)\right) \geq \frac{a^{*}(\bar{w}-w)}{1+a^{*}(\bar{w}-w)}$. The implication is that $\frac{a^{* 2} \dddot{x}^{2}}{2}+a^{*} \dddot{x} \geq \frac{a^{* 2} \ddot{x}^{2}}{2}+a^{*} \ddot{x}$ and, since both $\ddot{x}$ and $\dddot{x}$ are non-negative, we obtain that $\dddot{x} \geq \ddot{x}$.

Proof of the Corollary: To see that the Corollary is true it suffices to notice that, as $\bar{w}$ goes to infinity, so does $\ddot{x}$, and this means that $\bar{x}=w-\underline{w}$ in Theorem 1 . Therefore, only the first condition from expression (1) applies.

\section{References}

Bleichrodt, H., J. Doctor, Y. Gao, C. Li, D. Meeker and P. Wakker. "Resolving Rabin's Paradox." Manuscript, (2017).

Cox, J. and V. Sadiraj. "Small- and large-stakes risk aversion: Implications of concavity calibration for decision theory." Games and Economic Behavior 56 (2006): 4560.

Cox, J., V. Sadiraj, B. Vogt and U. Dasgupta. "Is there a plausible theory for decision under risk? A dual calibration critique." Economic Theory 54 (2013): 305333.

Palacios-Huerta, I. and R. Serrano. "Rejecting small gambles under expected utility." Economics Letters 91 (2006): 250-259.

Rabin, M. "Risk aversion and expected utility: A calibration theorem." Econometrica 68 (2000): 1281-1292.

Rabin, M. and R. Thaler. "Anomalies. Risk aversion." Fournal of Economic Perspectives 15 (2001): 219-232.

Rubinstein A. "Dilemmas of an economic theorist." Econometrica 74 (2006): 865883. 


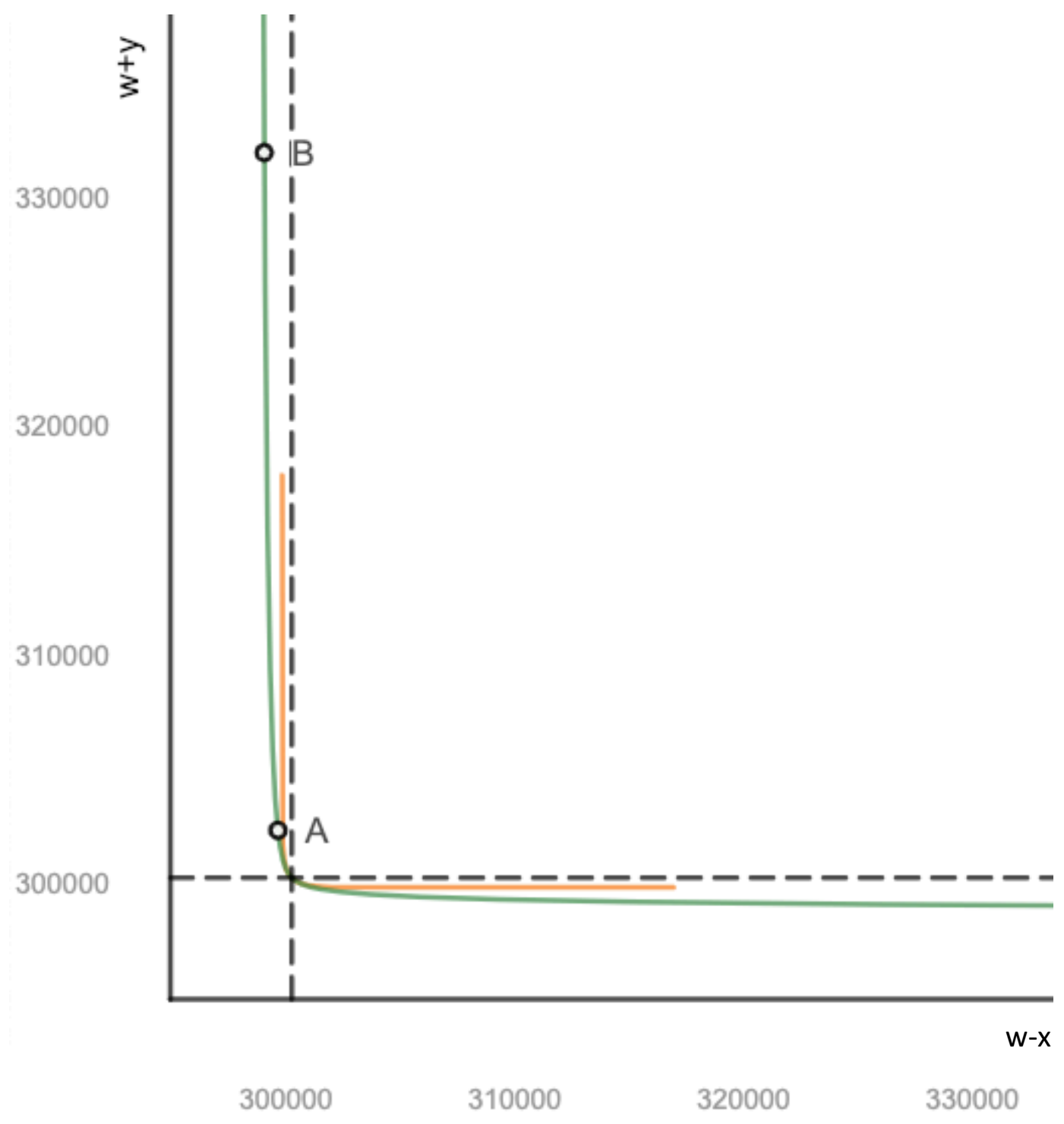

Figure 1. Carly rejects a 50-50 lose $\$ 100 /$ gain $\$ 120$ for all wealth levels. The orange indifference curve denotes the preferences of the CARA individual who is indifferent between taking and not taking such gamble. According to the Corollary, Carly is at least as risk averse as the individual with preferences represented by the green indifference curve. 